\title{
Observation and Modeling of the Solar-Cycle Variation of the Meridional Flow
}

\author{
Laurent Gizon • Matthias Rempel
}

Received: 7 September 2007 / Accepted: 3 March 2008 / Published online: 30 March 2008

(C) The Author(s) 2008. This article is published with open access at Springerlink.com

\begin{abstract}
We present independent observations of the solar-cycle variation of flows near the solar surface and at a depth of about $60 \mathrm{Mm}$, in the latitude range $\pm 45^{\circ}$. We show that the time-varying components of the meridional flow at these two depths have opposite sign, whereas the time-varying components of the zonal flow are in phase. This is in agreement with previous results. We then investigate whether the observations are consistent with a theoretical model of solar-cycle-dependent meridional circulation based on a flux-transport dynamo combined with a geostrophic flow caused by increased radiative loss in the active region belt (the only existing quantitative model). We find that the model and the data are in qualitative agreement, although the amplitude of the solar-cycle variation of the meridional flow at $60 \mathrm{Mm}$ is underestimated by the model.
\end{abstract}

Keywords Solar cycle: Models - Solar cycle: Observations - Velocity fields: Interior · Interior: Convective zone · Helioseismology: Observations · Magnetic fields: Models · Oscillations: Solar · Active regions · Supergranulation

\section{Introduction}

Solar oscillations are a unique tool to infer conditions inside the Sun. They have been recorded with great precision since 1996 with the Michelson Doppler Imager (MDI, Scherrer et al., 1995) onboard the Solar and Heliospheric Observatory (SOHO). Large-scale rotation inside the Sun can be estimated by inversion of the frequencies of millions of global

Helioseismology, Asteroseismology, and MHD Connections

Guest Editors: Laurent Gizon and Paul Cally.

L. Gizon $(\varangle)$

Max-Planck-Institut für Sonnensystemforschung, 37191 Katlenburg-Lindau, Germany

e-mail: gizon@mps.mpg.de

M. Rempel

High Altitude Observatory, National Center for Atmospheric Research, P.O. Box 3000, Boulder, CO 80307, USA 
modes of oscillation (e.g., Schou et al., 1998). Rotation is known to vary with time in the solar interior at the level of about $\pm 10 \mathrm{~m} \mathrm{~s}^{-1}$ (e.g., Schou, 1999; Howe et al., 2000, 2006a, 2006b; Vorontsov et al., 2002). These variations, known as torsional oscillations, consist of bands of faster and slower rotation that migrate in latitude as the 11-year solar magnetic cycle develops. Torsional oscillations may be driven by the Lorentz force owing to a dynamo wave (Schüssler, 1981; Yoshimura, 1981; Covas et al., 2000). Other explanations have been proposed (see Shibahashi, 2004, and references therein), including the suggestion by Spruit (2003) that torsional oscillations are driven by horizontal pressure gradients caused by photospheric magnetic activity.

Long time averages of surface Doppler measurements have shown the existence of a flow from the equator to the poles with an amplitude of $10-20 \mathrm{~m} \mathrm{~s}^{-1}$. An introduction to the theory of solar meridional circulation is provided by Shibahashi (2007). Temporal variations in the meridional flow have been reported by several authors. By tracking the small photospheric magnetic features, Komm, Howard, and Harvey (1993b), Komm (1994), and Meunier (1999) found a significant change in the meridional flow near sunspot latitudes, implying a solar-cycle variation. Variations in the surface Doppler meridional velocity have been detected by Ulrich and Boyden (2005), in particular at latitudes above $60^{\circ}$.

Local helioseismology (see, e.g., Gizon and Birch, 2005) also provides reasonable measurements of the meridional circulation for latitudes below about $50^{\circ}$ (Giles et al., 1997). The time-varying component of the meridional flow with respect to a long-term average does not exceed $\pm 5 \mathrm{~m} \mathrm{~s}^{-1}$ and is consistent with a small near-surface inflow toward active latitudes (Basu and Antia, 2003; Gizon, 2004; Zhao and Kosovichev, 2004; González Hernández et al., 2006; Komm, Howe, and Hill, 2006) and an outflow from active latitudes at depths greater than $20 \mathrm{Mm}$ (Chou and Dai, 2001; Beck, Gizon, and Duvall, 2002; Chou and Ladenkov, 2005). As discussed by Gizon (2004), these variations would appear to be caused by localized flows around localized regions of magnetic activity (Gizon, Duvall, and Larsen, 2001; Haber et al., 2001; Haber et al., 2004).

The purpose of this paper is twofold. First, we provide independent measurements of the temporal variations of the meridional circulation near the solar surface and at a depth of about $60 \mathrm{Mm}$. For the near-surface meridional flow, we use an original technique, which consists of measuring the advection of the supergranulation pattern (Gizon, Duvall, and Schou, 2003). The deeper meridional flow is calibrated from an earlier time-distance helioseismology observation by Beck, Gizon, and Duvall (2002). The meridional circulation measurements at these two depths are compared, in particular by looking at the 11-year periodicity of the flows (Section 2). Our study of meridional circulation confirms previous observations (as listed earlier) and is complementary to the analysis of zonal flows by Howe et al. (2006a).

Second, we wish to compare the observations with a theoretical model (Rempel, 2005; Rempel, Dikpati, and MacGregor, 2005; Rempel, 2006) based on a flux-transport dynamo combined with a geostrophic flow caused by increased radiative loss in the active region belt, according to Spruit's (2003) original idea. Section 3 provides a description of the model with a focus on meridional flows, since this aspect of the model has not been discussed elsewhere in detail. To our knowledge, this model is the only existing quantitative model of the solar-cycle dependence of internal flows: It is natural to ask whether it is consistent with the observations. The comparison between the observations and the model (Section 4) is encouraging, although some inconsistencies cannot be ignored. 


\section{Observations of the Meridional Flow}

\subsection{Near-Surface Layers}

The method we employ to infer flows near the solar surface is based on the analysis of Gizon, Duvall, and Schou (2003), which was originally applied to a single data set from 1996. Here we use a series of MDI full-disk Doppler images covering the period 1996-2002. Each year, two to three months of continuous Dopplergrams are available for analysis (MDI Dynamics runs). The MDI data after 2002 were not used simply because we are analyzing an existing preprocessed data set.

Dopplergrams were tracked at the Carrington rotational velocity to remove the main component of rotation. We used $f$-mode time - distance helioseismology (Duvall and Gizon, 2000 ) to obtain every 12 hours a $120^{\circ} \times 120^{\circ}$ map of the horizontal divergence of the flow field $1 \mathrm{Mm}$ below the photosphere. The main component of the divergence signal is due to supergranulation. For any given target latitude $(\lambda)$ we considered a longitudinal section of the data $10^{\circ}$ wide in latitude. Using a local plane-parallel approximation in the neighborhood of latitude $\lambda$, we interpolated the divergence signal onto a Cartesian grid sampled at $2.92 \mathrm{Mm}$ in the $x$ (prograde) and $y$ (northward) coordinates. The divergence signal was decomposed into its harmonic components $\exp \left[\mathrm{i}\left(k_{x} x+k_{y} y-\omega t\right)\right]$ to obtain a local power spectrum $P(\mathbf{k}, \omega ; \lambda)$, where $\mathbf{k}=\left(k_{x}, k_{y}\right)$ is the horizontal wavevector and $\omega$ is the angular frequency. At fixed $k R_{\odot}=120$, we fit for two functions ( $f$ and $g$ ) and a horizontal vector $(\mathbf{v})$ such that $P(\mathbf{k}, \omega ; \lambda)=f(\mathbf{k}) g[\omega-\mathbf{k} \cdot \mathbf{v}(\lambda)]$. This representation fits the data adequately. As was done by Gizon, Duvall, and Schou (2003), we interpret $\mathbf{v}(\lambda)$ to be a horizontal flow causing a Doppler shift $\Delta \omega=\mathbf{k} \cdot \mathbf{v}$. This flow is likely to be an average over the supergranulation layer, which has been estimated to reach depths greater than $10 \mathrm{Mm}$ by Zhao and Kosovichev (2003).

Figure 1 is a plot of $v_{x}(\lambda)$ and $v_{y}(\lambda)$ for each full-disk MDI run as a function of latitude in the range $|\lambda|<50^{\circ}$. To reduce random noise, the North - South symmetric component of $v_{x}$ and the antisymmetric component of $v_{y}$ are extracted. Over the period 1996-2002, $v_{x}$ varies by $12 \mathrm{~m} \mathrm{~s}^{-1}$ peak-to-peak at the equator (Figure 1(a)). The meridional flow is poleward with a mean amplitude of $10 \mathrm{~m} \mathrm{~s}^{-1}$ at latitude $20^{\circ}$ (Figure 1(b)). The peak-to-peak variation of the meridional flow is $7 \mathrm{~m} \mathrm{~s}^{-1}$ at $\lambda=30^{\circ}$ (i.e., a significant fraction of the time-average value). We estimate that the standard deviation of the noise at a particular latitude $\left(5^{\circ} \mathrm{bin}\right)$ for any given year is less than $1 \mathrm{~m} \mathrm{~s}^{-1}$. The systematic errors that depend on position on the solar disk have been measured to be very low (less than $5 \mathrm{~m} \mathrm{~s}^{-1}$ over the $120^{\circ} \times 120^{\circ}$ region of analysis).

\subsection{Deeper Inside the Sun}

To probe deeper layers in the solar convection zone, we used acoustic waves and timedistance helioseismology. For each three-month period, travel times were measured by cross-correlation of the Doppler oscillation signal recorded during the MDI structure program (offering nearly continuous coverage but lower spatial resolution) according to the procedure described by Giles (1999). Using a mean travel distance of $17^{\circ}$ enables us to probe layers about $60 \mathrm{Mm}$ below the surface. The full details of this analysis can be found in Beck, Gizon, and Duvall (2002). Waves that propagate in the North-South direction are used to infer the meridional flow, whereas waves that propagate East - West are used to infer the zonal flows. To convert travel-time shifts into flows in units of meters per second, we use a simple calibration of $v_{x}$ at a depth based on the observation by Howe et al. (2006a) 

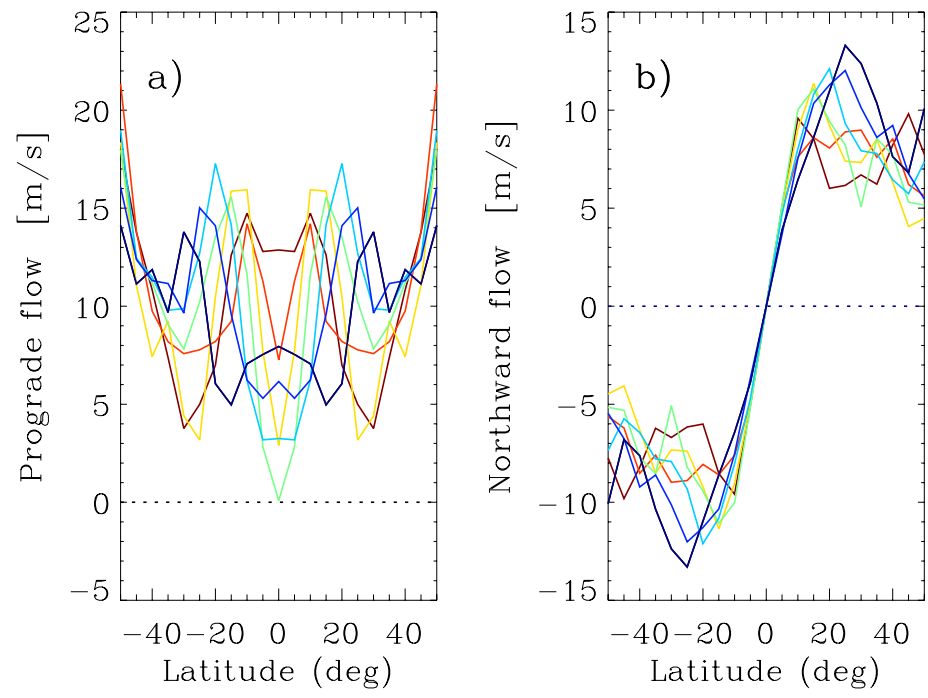

Figure 1 (a) Rotational velocity $\left(v_{x}\right)$ and (b) meridional flow $\left(v_{y}\right)$ near the solar surface as a function of latitude $(\lambda)$. Each MDI dynamics run is plotted with a different color from blue in 1996 to red in 2002 . The rotational velocity is given with respect to the rotational velocity of the small magnetic features (Komm, Howard, and Harvey, 1993a).

(global-mode helioseismology) that the amplitude of the time-varying component of the zonal flow is nearly independent of depth. We choose the near-surface zonal-flow measurements of Section 2.1 as a reference. The calibration of $v_{x}$ is then used to calibrate $v_{y}$. We find that the meridional flow at a depth of $60 \mathrm{Mm}$ is poleward at all latitudes and has a maximum value of $6 \mathrm{~m} \mathrm{~s}^{-1}$ at latitude $25^{\circ}$. For a particular year and at fixed latitude ( $\left.5^{\circ} \mathrm{bin}\right)$, the standard deviation of the noise is about $2 \mathrm{~m} \mathrm{~s}^{-1}$, significantly more than for the near-surface measurements.

\subsection{Solar-Cycle Variations}

To discuss the solar-cycle dependence of the flows and to study the phase relationship between the flows measured at the two different depths, we extract the 11-year periodic component from the data, as was done by Vorontsov et al. (2002) and Howe et al. (2006a) for zonal flows. At each latitude $\lambda$ and for each depth, we fit a function of the form

$$
\tilde{v}_{i}(\lambda, t)=\bar{v}_{i}(\lambda)+v_{i}^{\prime}(\lambda) \cos \left(\frac{2 \pi t}{11 \mathrm{yr}}+\phi_{i}(\lambda)\right)
$$

to the observed velocity $v_{i}(\lambda, t)$, where the index $i$ refers to either the $x$ or the $y$ component of the flow. The long-term average is given by $\bar{v}_{i}$, and the amplitude and the phase of the time-varying component are denoted by $v_{i}^{\prime}$ and $\phi_{i}$, respectively. We extract an 11-year periodicity from the data, since it is known from other observations that this is the dominant mode. (We do not determine the 11-year periodicity based on the data set itself.) Shorter and longer periodicities are certainly present in the data; however, the length of the data set does not allow for a determination of the full spectrum of modes.

The 11-year periodic components of the meridional and zonal flows are shown in Figure 2. The torsional-oscillation pattern is clearly seen at both depths with an amplitude and a 

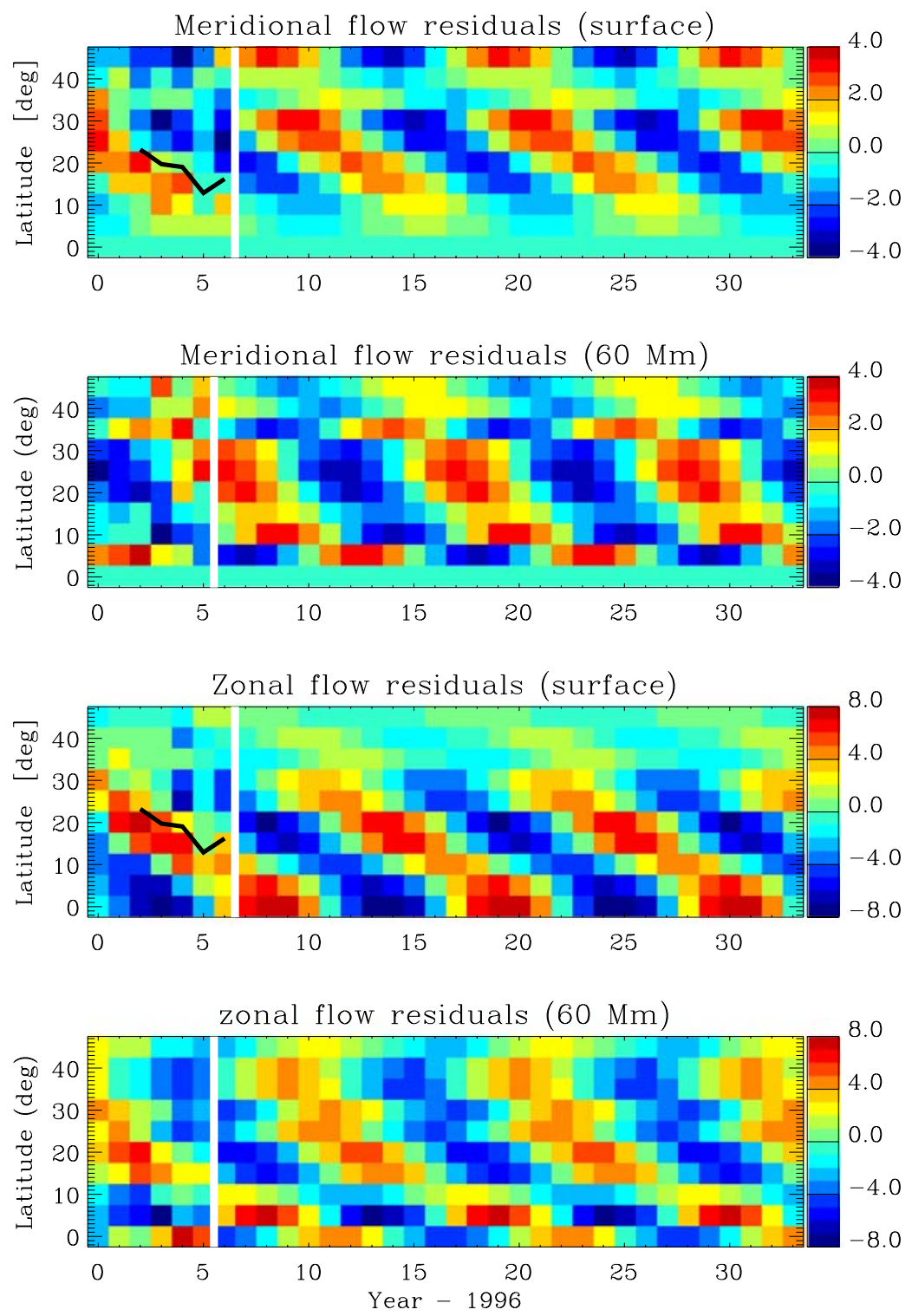

Figure 2 Eleven-year periodic component of the meridional (top two panels) and zonal (bottom two panels) flows as function of time and latitude at two different depths in the solar interior: near the surface (top and third panels) and $60 \mathrm{Mm}$ deep (second and bottom panel). The color bar is in units of $\mathrm{m} \mathrm{s}^{-1}$. A positive value indicates a poleward meridional or prograde zonal flow. The observations $\left(v_{i}-\bar{v}_{i}\right)$ cover the first six years, whereas the purely sinusoidal component $\left(\tilde{v}_{i}-\bar{v}_{i}\right)$ is extrapolated in time (beyond the white vertical white line). The black curves indicate the mean latitude of magnetic activity.

phase comparable to previous measurements (e.g., Howe et al., 2006a). The meridional flow also contains a significant 11-year periodic component. Near the solar surface, the residuals indicate the presence of a North - South inflow toward the mean latitude of activity (e.g., Zhao and Kosovichev, 2004; Komm, Howe, and Hill, 2006), but the data are consistent with 

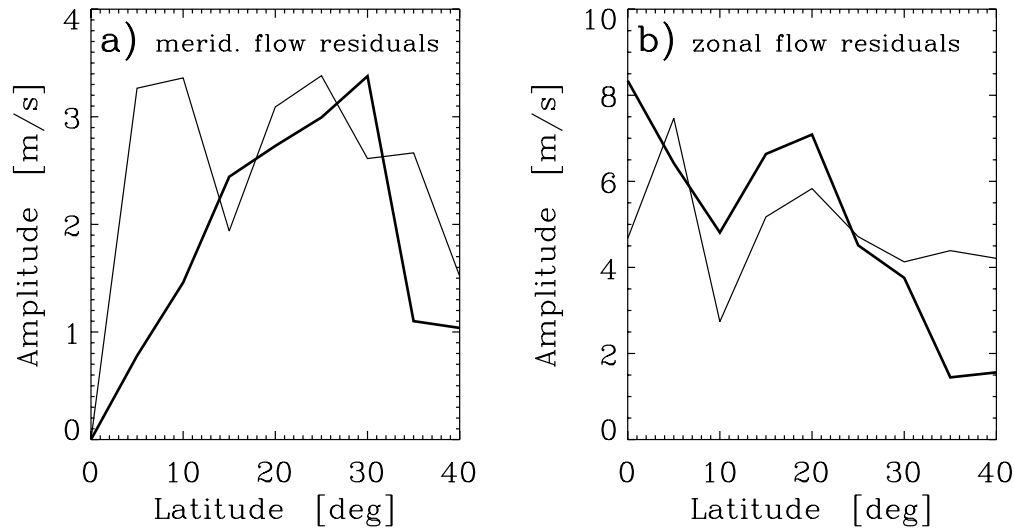

Figure 3 Amplitude $\left(v_{i}^{\prime}\right)$ of the 11-year periodic component of the (a) meridional and (b) zonal flows. The near-surface values (thick solid lines) are absolute measurements. The calibration of the observations at $60 \mathrm{Mm}$ depth (thin lines) follows from the assumption that the amplitude of the zonal torsional oscillation (panel (b)) is independent of depth over the latitude range $|\lambda|<45^{\circ}$.

Figure 4 Phase difference $[\Delta \phi=\phi($ deep $)-\phi($ surface $)]$ between the 11-year periodic components of the flows measured at a depth of $60 \mathrm{Mm}$ and near the surface. The solid line is for the meridional flow and the dashed line is for the zonal flow.

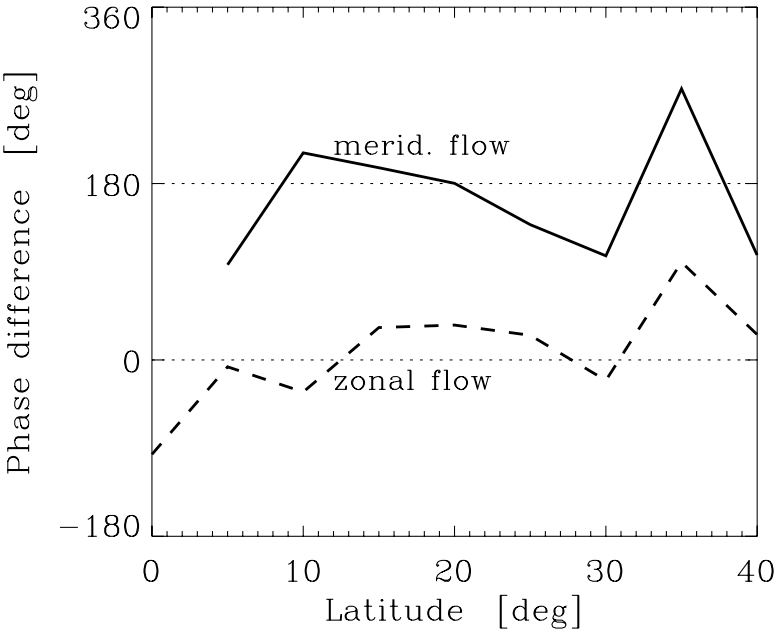

a horizontal outflow from the mean latitude of activity deeper into the convection zone (e.g., Chou and Dai, 2001; Beck, Gizon, and Duvall, 2002).

Figure 3 gives the amplitudes of the time-varying components of the flows $\left(v_{i}^{\prime}\right)$. Under the assumption (Section 2.2) that $v_{x}^{\prime}$ does not vary appreciably with depth $\left(5 \mathrm{~m} \mathrm{~s}^{-1}\right.$ latitudinal average), then the amplitude of the time-varying meridional flow $\left(v_{y}^{\prime}\right)$ is also found to be approximately independent of depth $\left(v_{y}^{\prime} \approx 3 \mathrm{~m} \mathrm{~s}^{-1}\right.$ at $20^{\circ}$ latitude). The evidence that the time-varying components of the meridional flow near the surface and deeper in the interior are anticorrelated is given in Figure 4, which shows the difference in $\phi_{y}$ at the two depths. In contrast, there is no significant phase variation with depth for the zonal flow. 


\section{Theoretical Model of Time-Varying Flows}

The model results presented here are based on a nonkinematic flux-transport dynamo model developed recently by Rempel. This model combines the differential rotation and meridional flow model of Rempel (2005) with a flux-transport dynamo similar to the models of Dikpati and Charbonneau (1999) and Dikpati and Gilman (2001). We emphasize that this model is intended to give a fundamental understanding of the basic cycle properties and their relation to observable variations of zonal and meridional flows. Therefore we focus here only on axisymmetric and North - South averaged quantities. Details of the model can be found in Rempel (2006). Since a detailed comparison with observed torsional oscillations can be found in Rempel (2006) and Howe et al. (2006b), we focus here on the meridional flow variations.

The differential rotation model utilizes a mean-field Reynolds-stress approach that parametrizes the turbulent angular momentum transport (Kitchatinov and Rüdiger, 1993; $\Lambda$-effect) leading to the observed equatorial acceleration. In this model the tachocline is forced through a uniform rotation boundary condition at the lower boundary of the computational domain. A meridional circulation, as required for a flux-transport dynamo, follows self-consistently through the Coriolis force resulting from the differential rotation.

The computed differential rotation and meridional flow are used to advance the magnetic field in the flux-transport dynamo model, while the magnetic field is allowed to feed back through the mean-field Lorentz force $\langle\mathbf{J}\rangle \times\langle\mathbf{B}\rangle$ (where the contribution of the fluctuating part $\left\langle\mathbf{J}^{\prime} \times \mathbf{B}^{\prime}\right\rangle$ is not well known and so is neglected here).

We find in our model that the Lorentz-force feedback can only account for the poleward propagating branch of the torsional oscillations, whereas the equatorward propagating branch in latitudes beneath $30^{\circ}$ requires additional physics. Parametrizing the idea proposed by Spruit (2003) that the low-latitude torsional oscillation is a geostrophic flow caused by increased radiative loss in the active region belt (owing to small-scale magnetic flux) leads in our model to a surface oscillations pattern in good agreement with observations. To force a torsional oscillation with around $1 \mathrm{nHz}$ amplitude, a temperature variation of around $0.2 \mathrm{~K}$ is required. As a side effect, close to the surface (in our model at $r=0.985 R_{\odot}$ ) the cooling produces an inflow into the active region belt of around $2.3 \mathrm{~m} \mathrm{~s}^{-1}$.

We incorporated this process by adding a surface-cooling term that is dependent on the toroidal-field strength at the base of the convection zone, which is assumed to be the source for active region magnetic field (the small-scale flux required for the surface-cooling being a consequence of the decay of active regions). Observations show that the low-latitude branch of torsional oscillations starts around one to two years before the sunspots of the new cycle appear. It is possible that magnetic flux rises toward the surface without forming sunspots in the beginning of a cycle, providing enough small-scale magnetic field; this is however currently neither confirmed nor ruled out by observations. Alternative explanations for the low-latitude branch of torsional oscillations such as the models of Schüssler (1981), Yoshimura (1981), and Covas et al. (2000) are based on the longitudinal component of the Lorentz force. Recently, Rempel (2007) showed that torsional oscillations forced that way are close to the Taylor - Proudman state (alignment of phase with the axis of rotation), which contradicts observations. In addition, the resulting meridional surface flow has the wrong sign (active region belt outflow). Despite some shortcomings, the model of Spruit (2003) is currently the only proposed explanation that is consistent with the observed meridional and zonal-flow variations close to the solar surface.

Figure 5 summarizes the results of the model in latitudes below $45^{\circ}$. Figure 5(a) shows the temperature fluctuation (color shades) caused by increased surface cooling in the active region belt. The contour lines indicate the magnetic butterfly diagram computed from 

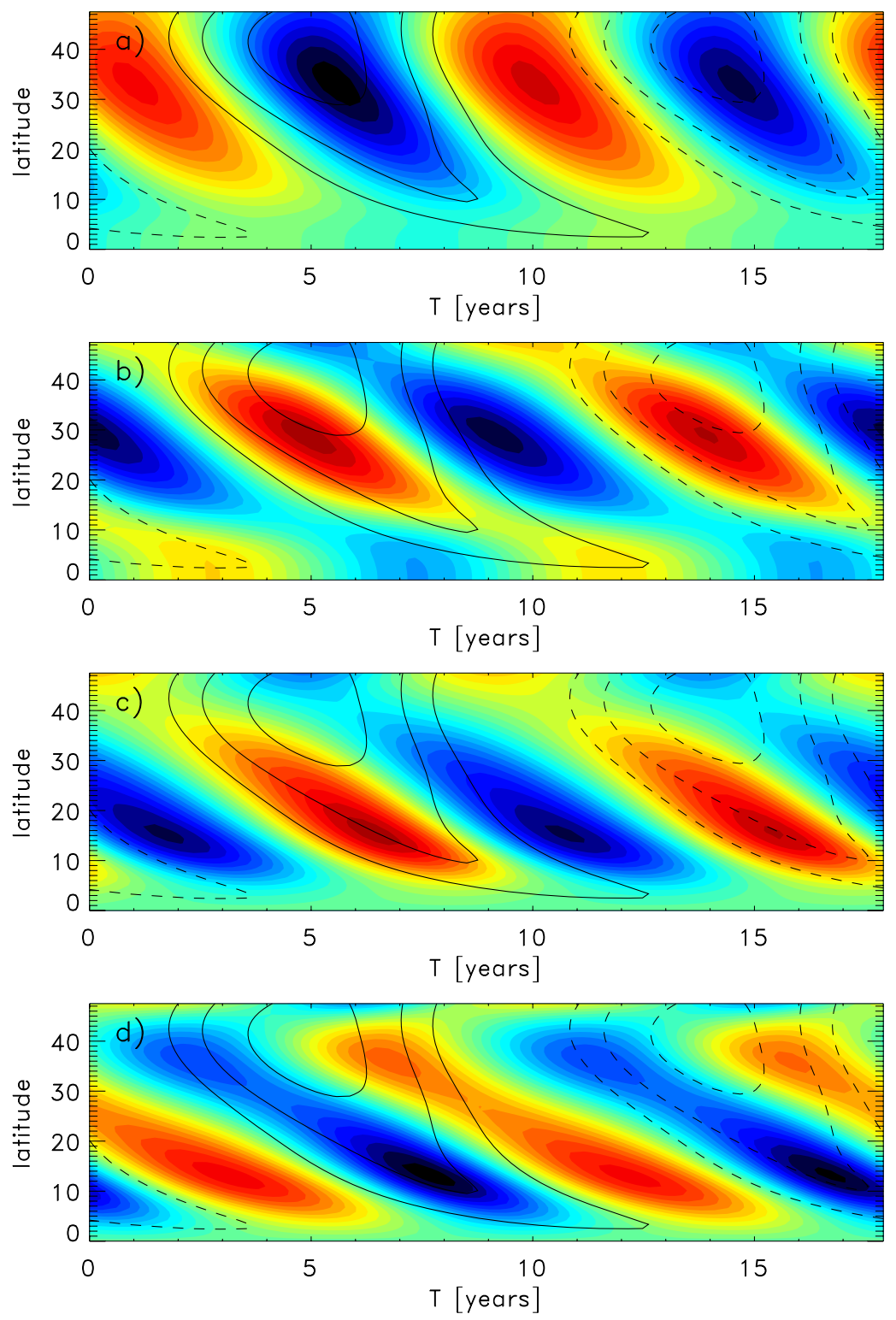

Figure 5 Model results. (a) Surface temperature variation (blue: cold, red: hot, amplitude: 0.2 K). (b) Torsional oscillations (blue: slower rotation, red: faster rotation, amplitude: $1.35 \mathrm{nHz}$ ). (c) Meridional flow variation at $r=0.985 R_{\odot}$ (blue: equatorward motion, red: poleward motion, amplitude: $2.3 \mathrm{~m} \mathrm{~s}^{-1}$. (d) Meridional flow variation at $r=0.93 R_{\odot}$ (blue: equatorward motion, red: poleward motion, amplitude: $0.22 \mathrm{~m} \mathrm{~s}^{-1}$ ). The variation of the meridional flow pattern at $r=0.985 R_{\odot}$ is almost in anticorrelation with the flow at $r=0.93 R_{\odot}(\approx 50 \mathrm{Mm}$ depth). In all four panels the contour lines indicate the butterfly diagram computed from the toroidal field at the base of the convection zone. 
the toroidal field at the base of the convection zone in the model. Figure 5(b) shows cycle variations of the angular velocity (torsional oscillations) and Figure 5(c) shows cycle variations of the horizontal meridional flow velocity. At the equatorward side of the active region belt (indicated by the butterfly diagram) the rotation rate is increased, which is consistent with the increased poleward meridional flow transporting material toward the axis of rotation. On the poleward side of the active region belt the rotation rate is lower, whereas the meridional-flow perturbation is equatorward. At a depth of around $50 \mathrm{Mm}$ (Figure 5(d)) the meridional-flow perturbation is almost anticorrelated to the surface flow (active region belt outflow), indicating that the surface cooling drives a flow system that closes in the upper third of the convection zone. The flow amplitude at a depth of $50 \mathrm{Mm}$ is around one order of magnitude lower compared to the surface flow owing to the significant increase in density.

\section{Discussion}

We presented a model that is qualitatively consistent with the observations, in particular with the phase of the solar-cycle variations of the flows. Since the surface-cooling term is parametrized, the model can only make predictions about the relative amplitude of zonal and meridional flows at different depths, and not about the absolute values. Near the surface, the model is in agreement with the data: The relative amplitudes of the torsional oscillation and the time-varying component of the meridional flow and their relative phase are reproduced well. Deeper in the interior, it appears that the model underestimates the amplitude of the time variations of the meridional flow by an order of magnitude; however, the flow variation is in antiphase to the surface flow as seen in the data. The lower velocity at depth in the dynamo model is a consequence of mass conservation (strong increase in density with depth). The much larger outflow that is observed in the data cannot be balanced by an inflow close to the surface unless the outflow is confined to a very narrow layer.

Overall, it is fair to say that the model is encouraging. Alternative models explaining low-latitude torsional oscillations through the action of the longitudinal Lorentz force tend to produce zonal flow patterns in the Taylor-Proudman state (Rempel, 2007) and meridional outflows rather than inflows close to the surface. However, zonal flows appear during solar minimum when no active regions are present. This requires, in the case of thermal forcing, that a sufficient amount of magnetic flux is present in the form of small-scale flux elements not evident in synoptic magnetograms (see, e.g., Section 6.3 of Spruit, 2003). Also a local treatment of the regions of strong magnetic-field concentrations (sunspots and active regions) might be necessary to obtain a better match between the model and the data.

On the observational side, it would be useful to invert the travel-time measurements to obtain improved and more reliable estimates of the depth variations of the flows.

Acknowledgements The National Center for Atmospheric Research is sponsored by the National Science Foundation. M. Rempel thanks Prof. Schüssler and the Max-Planck-Institut für Sonnensystemforschung for their hospitality.

Open Access This article is distributed under the terms of the Creative Commons Attribution Noncommercial License which permits any noncommercial use, distribution, and reproduction in any medium, provided the original author(s) and source are credited.

\section{References}

Basu, S., Antia, H.M.: 2003, Astrophys. J. 585, 553. 
Beck, J.G., Gizon, L., Duvall, T.L.: 2002, Astrophys. J. 575, L47.

Chou, D.-Y., Dai, D.: 2001, Astrophys. J. 559, L175.

Chou, D.-Y., Ladenkov, O.: 2005, Astrophys. J. 630, 1206.

Covas, E., Tavakol, R., Moss, D., Tworkowski, A.: 2000, Astron. Astrophys. 360, L21.

Dikpati, M., Charbonneau, P.: 1999, Astrophys. J. 518, 508.

Dikpati, M., Gilman, P.A.: 2001, Astrophys. J. 559, 428.

Duvall, T.L., Gizon, L.: 2000, Solar Phys. 192, 177.

Giles, P.M.: 1999, Ph.D. Dissertation, Stanford University.

Giles, P.M., Duvall, T.L., Scherrer, P.H., Bogart, R.S.: 1997, Nature 390, 52.

Gizon, L.: 2004, Solar Phys. 224, 217.

Gizon, L., Birch, A.C.: 2005, Living Rev. Solar Phys. 2(6). http://www.livingreviews.org//rsp-2005-6.

Gizon, L., Duvall, T.L., Larsen, R.M.: 2001, In: Brekke, P., Fleck, B., Gurman, J.B. (eds.) Recent Insights into the Physics of the Sun and Heliosphere: Highlights from SOHO and other Space Missions CS-203, Astron. Soc. Pacific, San Francisco, 189.

Gizon, L., Duvall, T.L., Schou, J.: 2003, Nature 421, 43.

González Hernández, I., Komm, R., Hill, F., Howe, R., Corbard, T., Haber, D.A.: 2006, Astrophys. J. 638, 576.

Haber, D.A., Hindman, B.W., Toomre, J., Bogart, R.S., Hill, F.: 2001, In: Wilson, A. (ed.) Helio- and Asteroseismology at the Dawn of the Millennium SP-464, ESA, Noordwijk, 209.

Haber, D.A., Hindman, B.W., Toomre, J., Thompson, M.J.: 2004, Solar Phys. 220, 371.

Howe, R., Christensen-Dalsgaard, J., Hill, F., Komm, R.W., Larsen, R.M., Schou, J., Thompson, M.J., Toomre, J.: 2000, Astrophys. J. 533, L163.

Howe, R., Komm, R., Hill, F., Ulrich, R., Haber, D.A., Hindman, B.W., Schou, J., Thompson, M.J.: 2006a, Solar Phys. 235, 1.

Howe, R., Rempel, M., Christensen-Dalsgaard, J., Hill, F., Komm, R., Larsen, R.M., Schou, J., Thompson, M.J.: 2006b, Astrophys. J. 649, 1155.

Kitchatinov, L.L., Rüdiger, G.: 1993, Astron. Astrophys. 276, 96.

Komm, R.W.: 1994, Solar Phys. 149, 417.

Komm, R.W., Howard, R.F., Harvey, J.W.: 1993a, Solar Phys. 145, 1.

Komm, R.W., Howard, R.F., Harvey, J.W.: 1993b, Solar Phys. 147, 207.

Komm, R.W., Howe, R., Hill, F.: 2006, Adv. Space Res. 38, 845.

Meunier, N.: 1999, Astrophys. J. 527, 967.

Rempel, M.: 2005, Astrophys. J. 622, 1320.

Rempel, M.: 2006, Astrophys. J. 647, 662.

Rempel, M.: 2007, Astrophys. J. 655, 651.

Rempel, M., Dikpati, M., MacGregor, K.: 2005, In: Favata, F., Schmidt, J. (eds.) Proc. 13th Cool Stars Workshop SP-560, ESA, Noordwijk, 913.

Scherrer, P.H., Bogart, R.S., Bush, R.I., Hoeksema, J.T., Kosovichev, A.G., Schou, J., Rosenberg, W., Springer, L., Tarbell, T.D., Title, A., Wolfson, C.J., Zayer, I., the MDI Engineering Team: 1995, Solar Phys. 162, 129.

Schou, J.: 1999, Astrophys. J. 523, L181.

Schou, J., Antia, H.M., Basu, S., Bogart, R.S., Bush, R.I., Chitre, S.M., Christensen-Dalsgaard, J., DiMauro, M.P., Dziembowski, W.A., Eff-Darwich, A., Gough, D.O., Haber, D.A., Hoeksema, J.T., Howe, R., Korzennik, S.G., Kosovichev, A.G., Larsen, R.M., Pijpers, F.P., Scherrer, P.H., Sekii, T., Tarbell, T.D., Title, A.M., Thompson, M.J., Toomre, J.: 1998, Astrophys. J. 505, 390.

Schüssler, M.: 1981, Astron. Astrophys. 94, L17.

Shibahashi, H.: 2004, In: Stepanov, A.V., Benevolenskaya, E.E., Kosovichev, A.G. (eds.) Multi-Wavelength Investigations of Solar Activity, IAU Symp. 223, Cambridge University Press, Cambridge, 23.

Shibahashi, H.: 2007, Astron. Nachr. 328, 204.

Spruit, H.C.: 2003, Solar Phys. 213, 1.

Ulrich, R., Boyden, J.E.: 2005, Astrophys. J. 620, L123.

Vorontsov, S.V., Christensen-Dalsgaard, J., Schou, J., Strakhov, V.N., Thompson, M.J.: 2002, Science 296, 101.

Yoshimura, H.: 1981, Astrophys. J. 247, 1102.

Zhao, J., Kosovichev, A.G.: 2003, In: Sawaya-Lacoste, H. (ed.) Local and Global Helioseismology: The Present and Future SP-517, ESA, Noordwijk, 417.

Zhao, J., Kosovichev, A.G.: 2004, Astrophys. J. 603, 776. 